\title{
Nuevas tendencias en educación, juegos serios con tecnología móvil en Windows 10 Mobile
}

\section{New trends in education, serious Games on mobile technology with Windows 10 Mobile}

\author{
Diego Alejandro Guerrero Peña \\ Ms. en Ingeniería Informática, profesor asociado, \\ investigador Grupo de investigación Automática, \\ Electrónica y Ciencias Computacionales \\ Instituto Tecnológico Metropolitano - ITM \\ Medellín, Colombia \\ Correo electrónico: diegoguerrero@itm.edu.co \\ Adriana Guerrero Peña \\ Ms. en MBA, profesora asistente, \\ investigadora grupo Davinci, \\ Facultad de Ciencias Exactas y Aplicadas \\ Instituto Tecnológico Metropolitano - ITM \\ Medellín, Colombia \\ Correo electrónico: adrianaguerrero@itm.edu.co \\ Erika Tatiana Muñoz Serna \\ Ingeniera de sistemas(c), \\ tecnóloga en Sistemas de Información, \\ Integrante del semillero de Investigación en Desarrollo de Software del \\ Grupo de investigación Automática, Electrónica y Ciencias Computacionales \\ Instituto Tecnológico Metropolitano - ITM \\ Medellín, Colombia \\ Correo electrónico: erikamunoz96639@correo.itm.edu.co
}

Recibido 19 de agosto de 2016; aprobado 8 septiembre de 2016

Cómo citar este artículo:

Guerrero, D. A., Guerrero, A., y Muñoz, E.T. (2017). Nuevas tendencias en educación, juegos serios con tecnología móvil en Windows 10 Mobile. Espiral, Revista de Docencia e Investigación, 7(1), 61 - 72.

\section{Resumen}

Objetivo. El presente artículo de investigación tiene como objetivo examinar las investigaciones realizadas acerca de los juegos serios mediados por la tecnología móvil, enfocándose en el sistema operativo Windows 10 Mobile y a su vez verificar la influencia de estos en el aprendizaje, haciendo mayor énfasis en la enseñanza y asimilación de conocimientos del modelado y la ingeniería del software y así resaltar la existencia o carencia de estos en nuestro entorno.

Metodología. El proceso de análisis aplicado al estudio se dividió en 3 etapas:

- Búsqueda de información. Se indagó en sitios de libre acceso en la web y artículos publicados en revistas especializadas, sobre la utilización de juegos serios mediados por plataformas móviles, haciendo énfasis en el sistema operativo
Windows Mobile y su implementación en la enseñanza del modelado de software.

- Constitución del análisis. Se tomó toda la información relevante al tema de investigación y se estructuró el análisis acerca de las nuevas tendencias en educación dadas por los juegos serios sobre la plataforma móvil Windows Mobile.

- Conclusiones del análisis. Se procede a inferir sobre los resultados obtenidos.

Resultados. Los resultados obtenidos permiten vislumbrar vacíos en los métodos de enseñanza tradicionales, los cuales pueden ser solucionados a través de metodologías apoyadas en los juegos serios y la tecnología actual.

Este artículo es parte del proyecto "Aprendizaje del modelado de software usando juegos informáticos sobre entorno WEB; estudio de caso: diagrama de secuen- 
cia" del Instituto Tecnológico Metropolitano - ITM, continuación del proyecto "Enseñanza y aprendizaje del modelado de software a través de juegos serios. Caso: Diagramas de interacción" (Guerrero, Anaya y Trujillo, 2010).

Palabras clave: Tecnología móvil, Windows Mobile, ingeniería del software, juegos serios móviles.

\section{Abstract}

Objective. This research article aims to check the research on serious games for mobile devices, focusing on devices running Windows 10 Mobile OS, to study their influence on software modeling and engineering teaching and learning, as well as their existence or absence in our environment.

We expect this research to become a useful tool for the future development of this field, which we believe needs a larger learning choice based on the development of useful competences for everyday life and decision-making. This could represent a valuable starting point for developers and a solid base for educators willing to find innovative ways to reach their students, thus increasing their engagement and share of autonomous work.

Methodology. The analytic process applied on this study was structured in 3 stages:

- Data mining: Research on free access websites and specialized magazines for contents on mediated use of serious games on mobile devices, paying special attention to those dealing with Windows Mobile OS and its implantation on software modeling teaching.

- Analysis setting-up: Gathering all the information relevant to the topic and structuration of the analysis on the latest trends on serious games for Windows 10 mobile.

- Conclusions: Inference of the results from the research.

Results. The obtained results allowed us to detect serious gaps on traditional teaching methods, which can be solved through methodologies supported in the serious games and the current technology.

This article is part of the project "Software modeling learning using web-based computer games; Case study: sequence diagram" of the Instituto Tecnológico Metropolitano (ITM), continuation of the project "Software modeling teaching and learning using serious games; Case study: Interaction diagrams" (Guerrero, Anaya y Trujillo, 2010).

Keywords: Mobil technology, Windows mobile, software engineering, mobile serious games.

\section{Introducción}

Muchos autores han demostrado la dificultad que tienen los estudiantes en aprender conceptos, los cuales pueden ser asimilados de forma más conveniente a través de los juegos serios (Serious Games), permitiendo que estos puedan llegar a ser aplicados por ellos a situaciones cotidianas (Greenfield, 1996). Muestra que los juegos serios pueden contribuir con importantes objetivos de la enseñanza de la ciencia como el desarrollo del aprendizaje a través de la observación y prueba de hipótesis y simulaciones científicas, esto debido a que el jugador es llevado a través de una secuencia de actividades, que le ayudan a hacer frente a problemas relacionados entre sí y a realizar formulaciones e interpretaciones pertinentes que permiten mejorar esas habilidades.

Al mismo tiempo, los juegos serios tienen el potencial de combinar la colaboración y participación, puesto que los estudiantes interactúan con la máquina y a la vez con sus compañeros pero los juegos serios móviles, además de las anteriores ventajas, también fusionan la posibilidad de que el usuario sea capaz de jugar, aprender y moverse a través de lugares reales (Stone, 2005).

La literatura al ser encontrada en el proceso de investigación, permitirá realizar el análisis de la información relacionada con la implementación de juegos serios en la plataforma Windows Mobile 10 y así establecer cuál es el nivel en que se encuentra este campo, cuáles son sus falencias y fortalezas actuales y los aportes que pueden hacer a la educación. Analizando lo anterior se genera este artículo que es un producto del proyecto "Aprendizaje del modelado de software usando juegos informáticos".

\section{Métodos de la investigación}

\section{Etapa del análisis}

Etapa 1. Búsqueda de información. Se realizó una exhaustiva indagación a través de diversos 
motores de búsqueda, utilizando métodos activos, los cuales son controlados por la persona ejecutora, generando una búsqueda por patrones que relacionan el contenido del tema de interés, brindando acceso a bases de datos, sistemas de comentarios y diferentes páginas web.

El tipo de búsqueda utilizado fue secuencial, ya que se recurre al uso del buscador introduciendo las palabras claves, permitiendo extraer todo lo relevante en cuanto al tema de la utilización de juegos serios mediados por plataformas móviles, haciendo énfasis en el sistema operativo Windows Mobile y su implementación en la enseñanza del modelado de software. La búsqueda se clasificó de la siguiente manera: Juegos serios en la educación, Juegos serios, Modelado de software y Juegos diagramadores de UML en Windows Mobile 10.

Etapa 2. Constitución del análisis. Una vez terminada la búsqueda de información y de realizar la respectiva clasificación necesaria para los fines de este estudio, se tomó todo lo relevante al tema de investigación y se estructuró el análisis de una manera jerárquica, tomando primero los temas más afines y terminando con los de menor relación, pero que aportan detalles importantes acerca de las nuevas tendencias en educación dadas por los juegos serios sobre la plataforma móvil Windows Mobile.

La literatura encontrada, principalmente en sitios de libre acceso en la web, fue la más representativa y la que aportó más información que contribuyó a nutrir el análisis de los juegos serios sobre la enseñanza del modelado de software en Windows Mobile 10.

Etapa 3. Conclusiones del análisis. Luego de estructurar el análisis y temas relacionados con este trabajo se procede a inferir sobre los resultados obtenidos, con la finalidad de que permita comprobar o rechazar las hipótesis generadas inicialmente sobre las ventajas o desventajas de utilizar los juegos serios en la enseñanza.

\section{Enfoque educativo}

Es importante que en el área educativa se hable de la calidad de la educación, es decir, cuando los estudiantes alcanzan los objetivos propuestos y llegan a ser ciudadanos competentes, capaces de delimitar los problemas, de proponer sus soluciones y de adaptarse continuamente a las necesidades de cambio; para ello es necesario impulsar nuevas maneras de enseñar además, verificar que la didáctica utilizada realmente aporta resultados satisfactorios.

Cabe resaltar que a lo largo de la historia, la educación ha progresado y las referencias didácticas se han modernizado y se empieza a dar un enfoque de qué es lo que realmente se quiere enseñar a los estudiantes, por qué y cómo involucrarlos más en su proceso de enseñanza; entonces se empieza a contemplar las metodologías de enseñanza basadas en el juego para la educación formal y superior, por consiguiente, la educación de este nuevo milenio debe contribuir al desarrollo de formas innovadoras de alfabetización digital que propicien el pensamiento crítico, la resolución de problemas, el trabajo colaborativo y nuevas formas de expresión (Díaz, Queiruga y Fava, 2015).

Según la RAE"el juego se puede ver como un ejercicio recreativo sometido a reglas, en el cual se gana o se pierde". Otra definición enfocada a nuestros intereses podría ser la que nos ofrece Sid Meier, creador de la exitosa saga de juegos Civilization, que define el juego "como una serie de decisiones con sentido".

El juego tiene una característica que es importante mencionar y es que genera un enlace con el jugador y esto sin duda se debe aprovechar para crear conocimiento, pues las actividades que identificamos como de carácter lúdico pueden coexistir perfectamente con las de la vida cotidiana, sin que necesariamente se evidencie una separación clara entre las mismas. $Y$ es entonces cuando hablamos de juegos serios 
(serious games). Una definición rápida acerca de este término podría ser la descrita por Zyda "una competición mental que se realiza con un ordenador de acuerdo con unas normas específicas y que utiliza el entretenimiento para el entrenamiento de habilidades relacionadas con educación, salud, política y objetivos estratégicos de comunicación".

Debido a estas fortalezas que brindan los juegos serios, el mundo ha iniciado proyectos de educación, buscando obtener mayores habilidades en los jugadores al momento de enfrentarse a situaciones reales en el mundo real. Por ejemplo, en noviembre de 2010, un consorcio Europeo, empezó a trabajar en un enfoque de mercado laboral diferente, utilizando el proyecto de aprendizaje móvil basado en el juego, el cual se centraba fundamentalmente en mejorar el auto aprendizaje a través de juegos serios y de esta manera brindarle a los aprendices mayores habilidades que ayuden a evolucionar en mayor medida el mercado laboral; en este proyecto optaron por elegir Smartphone y Tablet debido a su versatilidad (Imbellone, Botte y Medaglia, 2015).

En la anterior experiencia, surge un nuevo concepto, que es Mobile Learning, una metodología de enseñanza y aprendizaje valiéndose del uso de pequeños dispositivos móviles, tales como teléfonos móviles, PDA, tabletas, PocketPC, iPod y todo dispositivo de mano que tenga alguna forma de conectividad inalámbrica. Este nuevo concepto se está convirtiendo en un elemento clave en el desarrollo de los juegos serios, debido a la tendencia actual hacia los dispositivos móviles que muestran un cambio cultural en la forma de aprender. También es importante resaltar que el aprendizaje móvil (M-Learning), permite a los aprendices tener acceso al material en cualquier tiempo y lugar a través de muchos dispositivos, es decir, una aplicación M-Learning puede permitir extraer, interpretar y adaptar las funcionalidades al contexto actual de uso (americalearningmedia, s.f.).
Tomando como base los anteriores conceptos, se busca, también, brindar al mercado laboral, ingenieros de sistemas cada vez más idóneos y capaces, que estén según las necesidades de este nicho de mercado, por esto este trabajo busca enfocarse en analizar las alternativas y proyectos en cuanto a este tema en diferentes partes del mundo.

Para ello, es importante analizar la plataforma que se considera sería la más viable; pero cuando se empieza a verificar los diferentes sistemas operativos utilizados, se advierte que hay una gran competitividad, así que después de ver ventajas y desventajas, se decide considerar el sistema Windows 10 Mobile, ya que este nuevo sistema operativo tiene numerosas características diferenciadoras, como mayor optimización, estabilidad y seguridad y la más importante de ellas, que las aplicaciones que se desarrollen serán universales, ya que funcionarán en millones de dispositivos, debido a que son compatibles con Windows 10 y en Windows 10 Mobile, adaptando la interfaz al tipo de dispositivo que se utilice, pero funcionando bajo el mismo código, por lo que programando una única vez, la misma aplicación funcionará en ordenadores, tabletas y Smartphones (Microsoftinsider, s.f.).

Todas estas características que benefician tanto al usuario como a los desarrolladores, son herramientas muy poderosas, que fusionadas de la manera adecuada con una excelente metodología de enseñanza, pueden brindar óptimos resultados en cuanto al objetivo de imbuir conocimiento que pueda ser aplicado a situaciones prácticas del día a día.

\section{Juegos serios en la educación}

Los juegos serios están tomando un rol importante en la enseñanza primaria, secundaria y universitaria, cada día, en muchas más escuelas y universidades se utilizan los juegos como una herramienta para que los estudiantes puedan aprender las cosas de forma más rápida y 


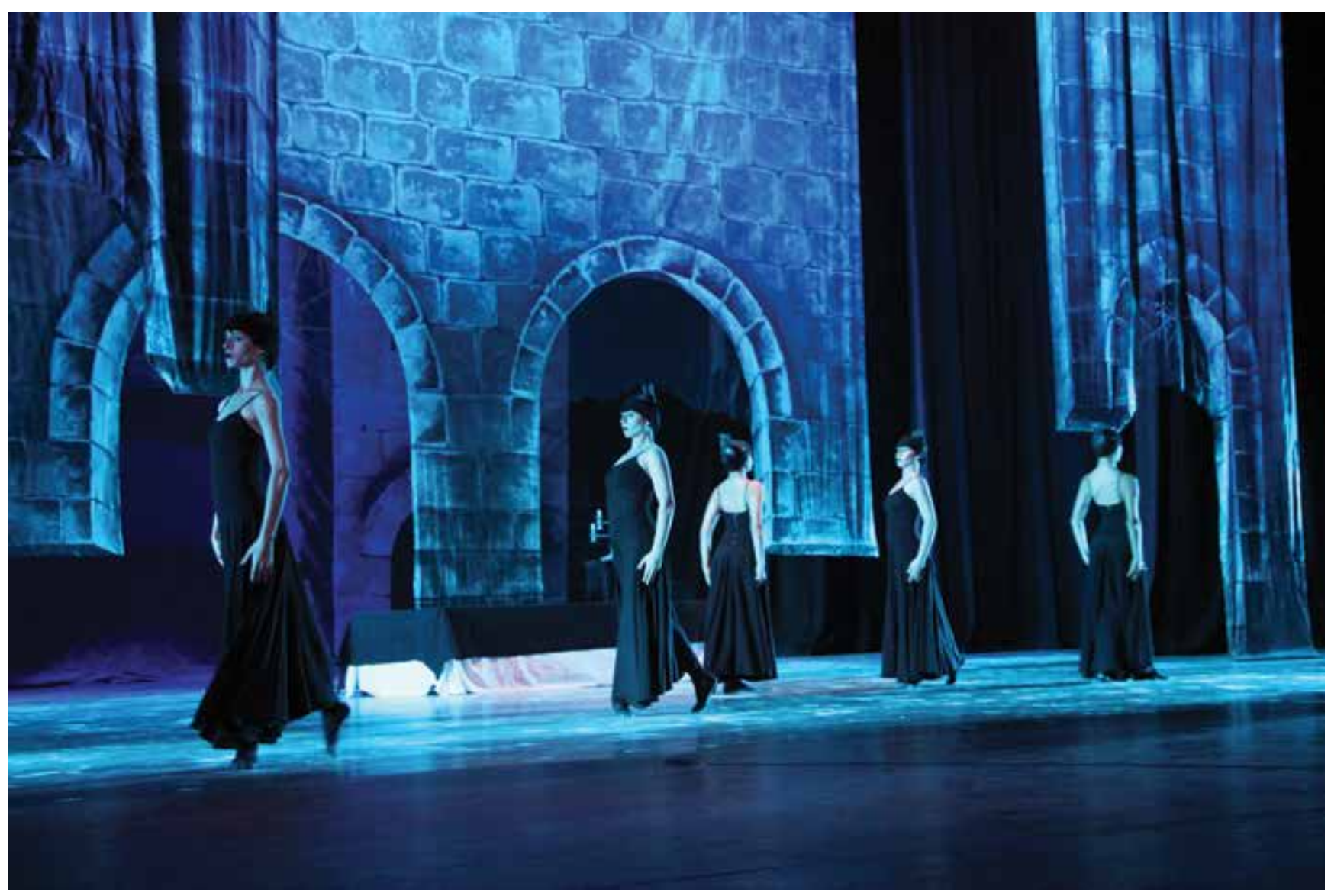

de una mejor manera (Gastón, 2014), además está probado científicamente que jugando aumentamos la producción de endorfinas que son estimuladoras y hace que las personas se sientan más capaces (Lorente y Pizarro, 2012).

La popularidad de los videojuegos, especialmente entre las personas jóvenes, hace de ellos un medio ideal para fines educativos (Falk, McLoughlin, Peters, Petridis y De Freitas, 2009), por esto, en los últimos años el mundo cada vez se interesa más en esta nueva forma de enseñar y esto no es ajeno a las empresas desarrolladoras de software ni a los grupos de investigación de las universidades, los cuales tienen la necesidad de brindar al mercado aplicaciones innovadoras y qué mejor que hacerlo a través de aplicaciones que ofrecen beneficios de aprendizaje mediante la diversión.

Se puede apreciar experimentos que muestran las ventajas de utilizar esta nueva forma de enseñanza, por ejemplo (Sandberg, Marinus y Geus, 2011) indican que realizan una prueba enseñando una segunda lengua a tres grupos de la escuela primaria, al primero le brindan una clase, al segundo le muestran una aplicación y le brindan la clase en una locación diferente y al tercero igual que al segundo, pero le permiten llevar la aplicación por quince días a sus casas y después de analizar los resultados indican que los estudiantes están motivados para usar la aplicación en su tiempo libre y que esto beneficia su aprendizaje.

Como muestra Piaget (1982), jugar y narrar son actividades cognitivas y afectivas que desde muy temprana edad posibilitan la expresión y desarrollo de la función simbólica, aportando contenidos y significados desde los entornos socioculturales en los cuales se sitúa el sujeto en un interjuego permanente con los significados y contenidos intrasubjetivos. Esto, entonces lleva a pensar que jugar y aprender están 
fuertemente ligados desde nuestra naturaleza humana.

También es importante indicar que los videojuegos expresan información semántica que configura un universo de sentido, por lo cual los jugadores aprenderán a partir de sus propios mundos posibles (Esnaola, 2004), mejorando la creatividad e innovación de cada jugador. Por otro lado, los juegos educativos se perciben con un alto potencial educativo por su carácter motivador (Foreman, 2004).

Igualmente, Blanco y González (2008) indican que la razón por la cual los videojuegos atraen tanto, está en que poseen lo que en psicología se denomina "factores dinamizadores de nuestra conducta"; en otras palabras, los videojuegos poseen el suficiente atractivo o despiertan bastante motivación como para que los niños y jóvenes se sientan conectados a su dinámica interna.

Por tanto, es importante que los modelos educativos empiecen a revolucionar adaptándose a las nuevas dinámicas que muestra la tecnología de nuestra época, como dice Carvajal (2009) el objetivo principal de toda institución educativa es formar lo mejor posible a sus alumnos; para lograrlo, los modelos tradicionales de enseñanza deben abrirse a nuevas experiencias didácticas, aplicando nuevas tecnologías como los videojuegos, que ofrecen una alternativa de aprendizaje más atractiva para el alumno y con una gran capacidad de interacción con el conocimiento.

Además, se cuenta con un componente de investigación que confirma la eficacia del juego en la educación, Esnaola (2008) dice, que de acuerdo con las investigaciones psicopedagógicas desarrolladas en este campo, ha quedado suficientemente demostrada la intervención de esta modalidad de juego en los procesos de construcción de la identidad social y en los aprendizajes psicoafectivos y varios estudios han intentado probar empíricamente el impac- to educativo de dos propiedades importantes de los juegos serios, la multimodalidad y la interactividad. Los resultados indican que tanto la multimodalidad y la interactividad contribuyen en los resultados educativos de forma individual (Riaño, 2012).

Pero sin lugar a dudas, para el desarrollo correcto de la educación y aprendizaje a través de dispositivos móviles dependerá de los niveles de involucramiento de directivos y docentes de instituciones educativas, padres de familia y estudiantes (Información Gerencial, s.f.).

Además, actualmente los juegos serios han recibido considerable preocupación en el campo de la educación, gracias a sus enormes ventajas respecto a metodologías anteriores de enseñanza, por tanto, se han formado comunidades de juegos educativos móviles en los últimos años y compañías como Apple ya cuentan con más de 65.000 aplicaciones educativas en el App Store de sus dispositivos.

Igualmente, el App store de Microsoft empieza a aumentar su oferta en esta categoría, en el momento cuenta con alrededor de 150 aplicaciones educativas - móviles, que no solo se pueden descargar en dispositivos móviles, sino también desde computadores y así aumentar su utilización.

Se pueden encontrar aplicaciones que son muy destacadas en esta categoría, como:

- SimCity: (Simcity, s.f.). Es un videojuego de construcción de ciudades desarrollado y publicado por la empresa Maxis, para las plataformas Microsoft Windows, Mac OS $X, y$ varias otras a lo largo de la serie, en este el jugador es el líder de una ciudad, el objetivo principal es hacerla crecer y prosperar, gestionando todos los aspectos de esta.

- Civilization: (Civilization, s.f.). Es un videojuego de estrategia por turnos, el cual dispone de un periodo de análisis antes de 
perpetrar la acción del juego, asegurando una separación entre el flujo del juego y el proceso de pensamiento, de manera que por turnos se llega presumiblemente a mejores soluciones, fue creado por Sid Meier y publicado por MicroProse en 1991 para las plataformas MS-DOS, Microsoft Windows, Apple Macintosh, Commodore Amiga, Atari ST y Super NES.

También se han desarrollado aplicaciones para la educación formal, tales como:

- PlayCode (Zhang y Lu, 2014). Desarrollada por la University of Huddersfield en Reino Unido, cuyos objetivos se enfocan en simplificar el aprendizaje de la programación, incluyendo el entretenimiento.

- SQL Island (Informatik, s.f.). Un juego de aventuras para aprender el lenguaje SQL de base de datos.

Es importante destacar que los juegos serios son aplicables en todas las áreas; solo basta con encontrar la necesidad, plantear el problema e iniciar el desarrollo del mismo y como dice (Tack, 2013) de alguna manera, el mundo de la educación va a atravesar uno de los cambios más masivos en los próximos cinco años de lo que ha visto en los últimos tres mil años.

\section{Juegos serios y el modelado de software}

La enseñanza de la ingeniería de software se ha realizado tradicionalmente con una combinación de clases expositivas y pequeños proyectos prácticos, pero las exigencias del mundo real obligan a combinar nuevas estrategias para comunicar estos conocimientos y la posibilidad de aplicar las metodologías activas para el desarrollo de las habilidades del siglo XXI, las cuales se cimientan en la potencialidad de generar entornos de aprendizaje ricos en recursos educativos, donde los aprendices puedan desarrollar proyectos y actividades que les permitan descubrir, aplicar y desarrollar el conocimiento (Marqués, 2001).
Como dice (Eldredge) en su estudio de Paradigmas y modelos pedagógicos, "cada momento histórico y cada cultura ha entendido la educación según el modelo de hombre buscado" y habla de tres modelos pedagógicos, se tiene la pedagogía tradicional, la cual busca lograr el conocimiento mediante la trasmisión de información, también se tiene la pedagogía activa, cuya prioridad está dada a la acción, la manipulación y contacto directo con los objetos y, por último, la pedagogía cognoscitiva, esta se basa en los postulados de la psicología genética, que estudia la génesis del conocimiento y propone el desarrollo del pensamiento y la creatividad como la finalidad de la educación, pero estas pedagogías deben complementarse, y una forma de hacerlo es mediante el juego.

Allí entran a formar parte importante los Juegos Serios para el aprendizaje del modelado de software, entre los cuales esta DesigMPS, diseñado para apoyar este proceso, también en la investigación realizada por (Guerrero, Guerrero y González, 2015) se tiene el juego Armar la Secuencia, con el cual se obtuvieron resultados que demuestran que los aplicativos de software, juegos serios, potencializan las estrategias educacionales de manera más adecuada, con respecto a la representación y comprensión conceptual de la competencia sobre diagramas de secuencia en el modelado de software, en comparación con los métodos tradicionales.

Otras aplicaciones diseñadas para este propósito son: Modela (Blanco, López y Viejo, 2015), es un juego serio donde los estudiantes de ingeniería de software, pueden aprender a modelar requerimientos con casos de uso, esta es una aplicación de escritorio; también se tiene $U M L$, que es una herramienta online, que permite crear los diagramas a partir de unos comandos escritos en texto plano, tiene diferentes tipos de diagramas, como de casos de uso, de clases y de actividad. 
Realizando el enfoque sobre la plataforma de nuestro interés, encontramos las siguientes aplicaciones en la tienda de aplicaciones de Microsoft, que son soportados por los sistemas operativos Windows 10 Mobile, Windows Phone 8.1, Windows Phone 8:

- Grapholite: es un diseñador para crear diagramas, dirigido a todo tipo de gráficos comerciales y dibujos técnicos y bocetos de documentos de aspecto profesional, complejos y simples.

- UML Pocket Reference (Microsoft, s.f.): es una aplicación para crear diagramas UML de forma rápida para los estudiantes e ingenieros de software.

- YUML (genbetadev, s.f.): la herramienta permite crear diagramas a partir de unos comandos escritos en texto plano y posee diferentes tipos, como, de casos de uso, de clases y de actividad.

- UML Class Wizard (microsoft, s.f.): proporciona una forma fácil de usar herramientas para crear varios tipos de diagramas como de Flujo, la red de ordenadores y de casos de uso.

- UML Pro (microsoft, s.f.): es la herramienta principal de diagramación UML de Windows 8. Especialmente diseñado para trabajar con dispositivos touch.

- Flowdia Diagrams: es una herramienta fácil de usar, permite crear diagramas de flujo profesionales de calidad, mapas mentales, diagramas BPMN 2.0, Red y diseños de servidor, diagramas UML, rack de actividad y diagramas de clases.

- UML Diagram PRO: esta aplicación le permite crear algunos diagramas UML.

- UML Diagram Generator Suite: es una herramienta muy útil para el diseño de un software. Esta aplicación, genera diagramas de casos de uso y el uso extendido de diagramas de casos para cada funcionalidad.

- Sketchagram: es un editor de diagramas, que es compatible con varios tipos de diagramas.

- WP8WSD: le permite generar y guardar diagramas de secuencia UML usando el potente sistema (Tienda Aplicaciones Microsoft, s.f.).

En general todos estos juegos o aplicaciones permiten crear diagramas UML (casos de uso, secuencia, clase, etc.), pero no se complementan con una metodología lúdica de enseñanza, que le permita al estudiante interiorizar de una forma dinámica el conocimiento y así poderlo manipular para crear nuevas cosas a partir de este.

\section{Juegos serios en Colombia}

También es importante analizar la situación en este momento en Colombia, pues se está generando un fuerte impacto alrededor de toda esta dinámica y por esto el Ministerio de Educación y el Ministerio de las TIC realizaron el I Encuentro de Productores y Desarrolladores de Aplicaciones Educativas para Dispositivos Móviles (Ministerio de Educación, s.f.), en el que se plantearon importantes objetivos, como consolidar una red de aliados en el país, basada en la promoción de espacios de discusión en torno a la producción y gestión de una oferta de calidad de aplicaciones educativas.

Hay que destacar que en este momento existen algunas aplicaciones móviles educativas, como son las desarrolladas por la empresa 'Red Mapache' que podemos encontrar en https://es-la.facebook.com/RedMapache/, la cual desarrolla libros y juegos para dispositivos móviles que buscan ayudar a niños de 6 a 8 años para mejorar procesos de comprensión de lectura, también tiene otras aplicaciones como por ejemplo el juego llamado 'Shamanimals' que está enfocado en mejorar el vocabulario 


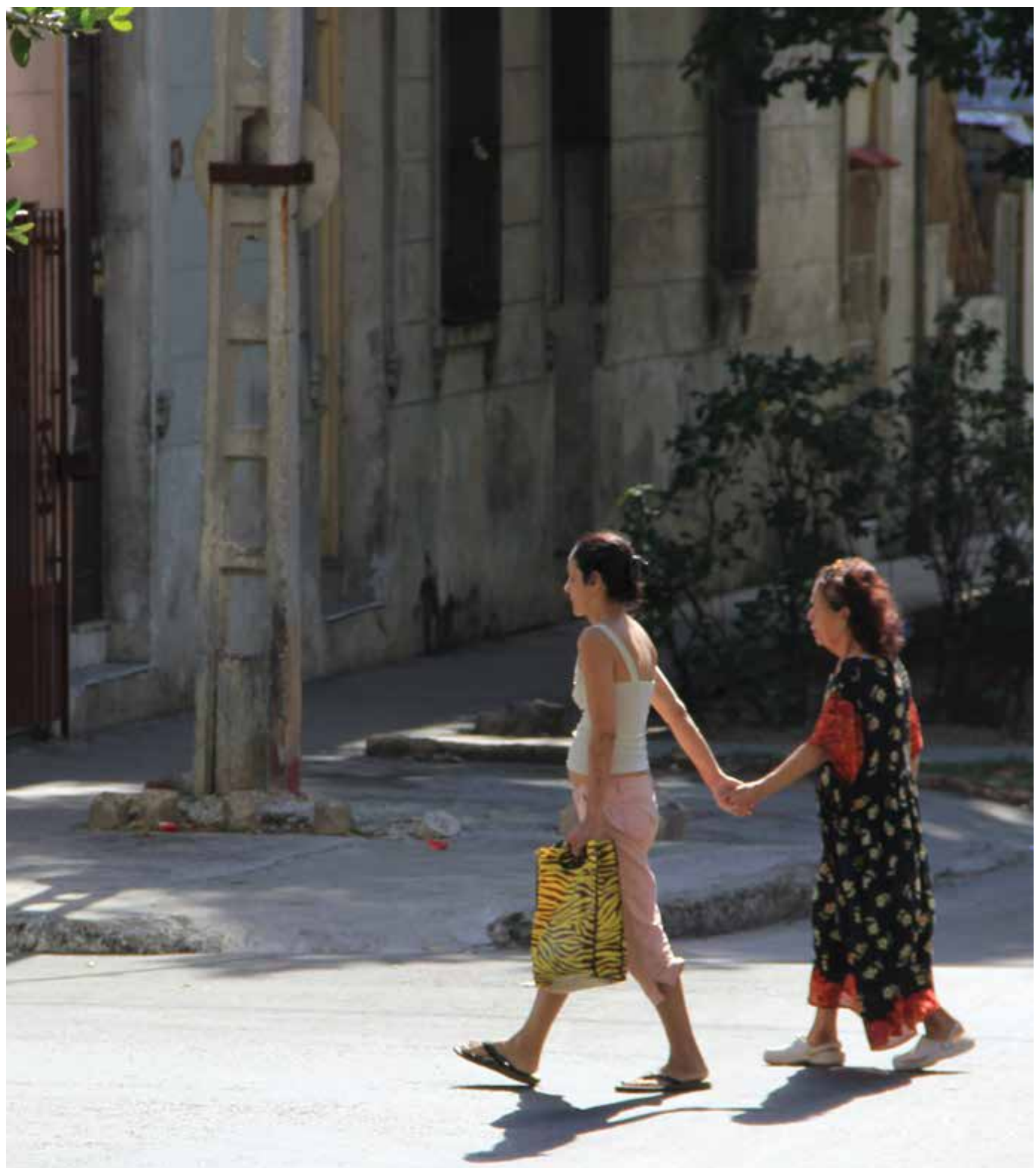


de los niños. Igualmente se cuenta con el portal educativo "Colombia aprende", que se puede encontrar en www.colombiaaprende.edu. co del Ministerio de Educación, quien cada vez busca incluir y ofrecer más aplicaciones enfocadas a mejorar los procesos de enseñanza.

Se puede inferir, que en Colombia se tiene un apoyo grande para avanzar en el ámbito de aplicaciones móviles, pero aún hay vacíos en aplicaciones para estudios universitarios y mucho más enfocados al área de sistemas; puesto que al realizar una búsqueda en esta área no hay información relevante que nos permita indicar que se estén dando desarrollos enfocados a la ingeniería de software.

\section{Conclusiones y trabajos futuros}

Desde el punto de vista de la mecánica de juego, uno de los conceptos más importantes es el "Flow", conseguir el estado en que el jugador encuentra un reto acorde a sus capacidades, de modo que no se frustre por la excesiva dificultad ni se aburra por lo escaso de esta (Riaño, 2012). En ambos casos el resultado es que el jugador perderá el interés en el juego.

Dado este principio, en el análisis de los juegos serios sobre modelado de software en Windows Mobile, se puede observar que hay carencia en aplicaciones móviles que logren cumplir las características necesarias para poder ser clasificadas entre juegos serios que efectivamente permitan mejorar los indicadores de entendimiento y asimilación del tema de ingeniería de software para sus estudiantes.

Además, se logró visualizar algunas ventajas y beneficios que se han logrado con esta metodología fusionada con la tecnología móvil y la importancia de generar nuevas alternativas en temas tan fundamentales en la ingeniería de sistemas como es el modelado de software.

También se pudo apreciar la penetración que ha logrado esta metodología en campos como la ciencia, la industria, la educación me- dia y superior y los excelentes resultados que se han logrado al utilizar tecnología móvil con juegos serios.

Para dar continuidad al progreso del trabajo se recomienda evolucionar el aplicativo mediado por la tecnología informática a equipos móviles, en el sistema operativo Windows Mobile, para que los estudiantes puedan interactuar en cualquier lugar y en cualquier momento, además de continuar con el desarrollo de juegos serios soportados pedagógicamente y didácticamente (Guerrero, Anaya y Trujillo, 2010).

\section{Reconocimiento}

El trabajo realizado tiene un gran componente formativo, de allí la participación del semillero de investigación en desarrollo de software del Grupo Automática, Electrónica y Ciencias Computacionales del Instituto Tecnológico Metropolitano ha sido muy importante, a sus integrantes nuestro agradecimiento.

\section{Referencias}

América Learning y Media. (s.f.). Recuperado de http:// www.americalearningmedia.com/edicion-009/105analisis/665-dispositivos-moviles-en-la-educacion

Blanco, F., González, C. (2008). Emociones con videojuegos: Incrementando la motivación para el aprendizaje. Recuperado de http://campus.usal.es/ teoriaeducacion/ rev_numero_09_03/n9_03_gonzalez_blanco.pdf

Blanco, S., López, F., Viejo, A. (2015). Juego educativo enfocado al desarrollo de habilidades para aprender a modelar requerimientos con artefactos UML. (Proyecto de Investigación). Recuperado de http://journal.seriousgamessociety.org/index.php?journal=IJSG\&page=article\&op=vi ew\&path\%5B\%5D=41\&path\%5B\%5D=pdf_7

Boyle, E., MacArthur, E., Hainey, T., y Boyle, J. (2011). A systematic literature review of empirical evidence on computer games and serious games. Recuperado de http://www.sciencedirect.com/science/article/pii/ S0360131512000619

Carvajal, D. (2009). ¿Pueden los videojuegos jugar un papel relevante en educación? Recuperado de https:// www.researchgate.net/profile/David_Carvajal/ publication/280490309_Pueden_los_videojuegos_jugar_un_papel_relevante_en_educacion/ links/55b62f1908ae092e9655c64c.pdf

Corporación Colombia Digital. Juegos serios: DragonBox, un ejemplo a seguir. Recuperado de http://colombiadigital. net/actualidad/experiencias/item/4944-juegos-seriosdragonbox 
Díaz, J., Queiruga, C., Fava, L. (2015). Juegos serios y educación. Recuperado de http://sedici.unlp.edu.ar/bitstream/handle/10915/46458/Documento_completo. pdf? sequence $=1$

Eldredge, G. (s.f.). Universidad Tecnológica Equinoccial. Recuperado de http://app.ute.edu.ec/portalintegrado/ login.aspx

Esnaola, G. (2004). Aprender a leer el mundo del siglo XXI a través de los videojuegos. Recuperado de http://www. ugr.es/ sevimeco/revistaeticanet/Numero3/Articulos/ Formateados/Aprender+a+leer\%5B1\%5D.pdf

Esnaola, G., y Levis, D. (2008). La narrativa en los videojuegos: un espacio cultural de aprendizaje socioemocional. Recuperado de http://campus.usal.es/ teoriaeducacion/ rev_numero_09_03/n9_03_esnaola_levis.pdf

Falk, E., McLoughlin, L., Peters, C., Petridis, P., De Freitas, S. (2009). Serious Games in cultural Heritage. Recuperado de http://eprints.bournemouth.ac.uk/20460/

Foreman, J. (2004). Gamed-Based Learning: How to Delight and Instruct in the 21st Century. EDUCASE Review.

Formación Gerencial. Recuperado de http://blog.formaciongerencial.com/2010/05/03/tecnologia-movil-en-laeducacion-aplicaciones-usos-y-tendencias/

GameLearn. Recuperado de: https:/game-learn.com/thefuture-of-serious-games-through-the-lens-of-mobiledevices/

Gastón, D. (2014). Juegos serios, evaluación de tecnologías y ámbitos de aplicación. Recuperado de: http://sedici. unlp.edu.ar/handle/10915/40330

Genbetadev. Recuperado de http://www.genbetadev.com/ herramientas/yu ml-herramienta-online-para-crear-diagramas-uml-a-partir-de-texto-plano

Génova, G. (s.f.). Conceptos básicos de modelado, cap. 1.

George, S., Serna, A. (2011). Introducing Mobility in Serious Games: Enhancing Situated and Collaborative Learning. Recuperado de: https://link.springer.com/chapter/10.1007/978-3-642-21619-0_2

Greenfield, P. M. (1996). Video games as cultural artifacts. Recuperado de https://www.researchgate.net/publication/232456983_Video_games_as_cultural_artifacts

Guenaga, M., Arranz, S., Aguilar, E., Ortiz, A., Rayón, A., Bezanilla, M., Menchaca, I. (2013). Serious Games para el desarrollo de competencias orientadas al empleo.

Guerrero, D., Anaya, R., Trujillo, J. (s.f.). Enseñanza y aprendizaje del modelado de software a través de juegos serios Caso: Diagramas de interacción.

Guerrero, D., Guerrero, A., González, P. (2015). Aplicativos de software juegos serios un cambio en la enseñanza y aprendizaje de las competencias del modelado de software.

Hildmann, R., Hirsch, B. (s.f.). Raising Awareness for Enviromental Issues Trough Mobile Device Based Serious Games.

Imbellone, A., Botte, B., Medaglia, C. (2015). Serious Games for Mobile Devices: the InTouch Project Case Study. Recuperado de http://journal.seriousgamessociety.org/index.php?journal=IJSG\&page=article\&op=view\&path\% $5 \mathrm{~B} \% 5 \mathrm{D}=41$

Informatik. (s.f.). Recuperado de: http://wwwlgis.informatik. uni-kl.de/cms/courses/informationssysteme/sqlisland/
Latinpost. (s.f.). Recuperado de http://www.latinospost. com/articles/31446/20131112/ios-vs-android-windows-phone-market-share-france.htm

Lorente, P., Pizarro, M. (2012). El juego en la enseñanza de español como lengua extranjera. Nuevas perspectivas. Recuperado de http://www.tonosdigital.es/ojs/index.php/ tonos/article/viewFile/821/554

Ma, M., Oikonomou, A., Jain, C.L. (2011). Serious Games and Edutainment Applications. Recuperado de https://link. springer.com/book/10.1007/978-1-4471-2161-9

Marqués, P. (2001). Didáctica. Los procesos de enseñanza y aprendizaje. La motivación. Recuperado de http:// dewey.uab.es/pmarques/actidid.html

Marsh, T. (2011). Serious Games continuum: Between games for purpose and experiential environments for purpose. Recuperado de http://www.sciencedirect.com/science/ article/pii/S1875952110000224

Microsoft Store. (s.f.). Recuperado de https://www.microsoft. com/en-us/store/apps/uml-class-wizard/9wzdncrfj8w4

Microsoft Store. (s.f.). Recuperado de https://www.microsoft.com/enus/store/apps/grapholite-diagrams-flowcharts-and-floor plans-designer/9wzdncrddgs5

Microsoft. (s.f.). Recuperado de https://www.microsoft.com /en- us/store/apps/uml-pocketreference/9nblggh0k93q

Microsoft. (s.f.). Recuperado de http://www.microsoftinsider.es/74269/por-que-windows-10-mobile-sera-superior-a-su-competencia/

Ministerio de Educación. (s.f.). I Encuentro de productores y desarrolladores de aplicaciones educativas para dispositivos móviles. Recuperado de http://www.mineducacion. gov.co/cvn/1665/w3-article-342111.html

Perdita, J. (s.f.). GUIDE: Games with UML for Interactive Design Exploration. Recuperado de http://www.sciencedirect. com/science/article/pii/S0950705107000548

Piaget, J. (1991). La formación del símbolo en el niño. Imitación, juego y sueño. Imagen y representación. Buenos Aires: Fondo de Cultura Económica. Recuperado de http://bloguamx.byethost10.com/wp-content/uploads/2015/04/ formacic2a6n-del-simbolo-piaget.pdf?i=2

Rayborn, E. (2005). Design and evaluation challenges of serious games. Recuperado de http://www.sandia.gov/ adaptive-training-systems/papers/p2049-raybourn.pdf

Real Academia Española. (s.f.). Recuperado de http://www. rae.es/

Revista Unam. (2009). Recuperado de http://www.revista. unam.mx/vol.10/num11/art79/int79.htm

Riaño, J. (2012). Investigación para la empleabilidad. Proyecto Serious Games. Recuperado de http://www.bizkailab. deusto.es/wp-ontent/uploads/2013/05/estado_arte_ SG4E.pdf

Romero, M. (2012). Serious Games para el desarrollo de las competencias del siglo XXI. Recuperado de http:// www.redalyc.org/articulo.oa?id $=54724753002$

Sánchez, J., y Olivares, R. (2011). Problem solving and collaboration using mobile serious games. Recuperado de http://www.sciencedirect.com/science/article/pii/ S0360131511000935 
Sandberg, J., Marinus, M., Geus, K. (2011). Mobile English learning: An evidence-based study with fifth Graders. Recuperado de http://www.sciencedirect.com/science/article/ pii/S0360131511000327

SimCity. (s.f.). Recuperado de http://www.simcity.com/

Stapleton, A. (2004). Serious Games: Serious Opportunities. Recuperado de http://www.autzones.com/din6000/textes/semaine12/Stapleton(2004).pdf

Stone, R. (2005). Serious Gaming - Virtual Reality's Saviour? Recuperado de https://www.researchgate.net/publication/228353462_Serious_Gaming-Virtual_Reality's_Saviour
Tack, D. (2013). Serious Games and the Future of Education. Recuperado de https://www.forbes.com/sites/danieltack/2013/09/12/serious-games-and-the-future-ofeducation/\#33eb1c8d679d

Universidad Tecnológica Equinoccial. (s.f.). Recuperado de http://app.ute.edu.ec/VideoConferencias/873/ PARADIGMAS_Y_MODELOS_PEDAGOGICOS.pdf

Wikipedia. (s.f.). Recuperado de https://es.wikipedia.org/ wiki/Aprendizaje_electr\%C3\%B3nico_m\%C3\%B3vil

Zhang, J., y Lu, J. (2014). Using Mobile Serious Games for Learning Programming. Recuperado de http://openaccess. uoc.edu/webapps/o2/bitstream/10609/40577/8/sblancopaTFM0115memoria.pdf 